\title{
The Vertical Plate in Laminar Free Convection: Effects of Leading and Trailing Edges and Discontinuous Temperature
}

By Arthur F. Messiter, Dept of Aerospace Engineering, University of Michigan, Ann Arbor, U.S.A., and Amable Liñán, Escuela Técnica Superior de Ingenieros Aeronáuticos, Madrid, Spain

\section{Introduction}

If a heated plate is oriented vertically in a fluid which is at rest and acted upon by gravitational forces, for large values of the Grashof number, the fluid moves upward along the plate in a thin boundary layer and then forms a thin plume above the plate. Early studies of this motion are discussed in the review articles by Ostrach [1] and Ede [2]. For a semi-infinite plate the equations for the laminar free-convection boundary layer have solutions of the similarity type, as noted by Schmidt and Beckmann [3] and Ostrach [4], who also gave numerical solutions of the corresponding ordinary differential equations. These analyses used the Boussinesq approximation, and therefore assumed small temperature changes.

In an effort to obtain a better approximation for a finite plate at moderate Grashof numbers, Yang and Jerger [5] looked for a perturbation solution of the Boussinesq equations, in terms of an inner expansion for the boundary layer along the plate and an outer expansion for the small external flow induced by the entrainment of fluid into the boundary layer. They assumed that the entrainment of fluid into the plume had negligible effect on the velocity just outside the boundary layer at the plate. It was noted that their expansions are singular at the leading edge, but the expression obtained for the second-order local heat transfer is integrable, and the corresponding correction to the average Nusselt number was estimated by integration along the plate. In a separate paper Yang [6] examined the plume development near the trailing edge and drew the conclusion that neglecting the effects of plume entrainment introduced no significant errors in the calculation of heat transfer from the plate. Hardwick and Levy [7] obtained a numerical solution of the Boussinesq equations for the trailing-edge region and noted an upstream influence of the trailing edge. A closely related problem was considered by Kelleher [8], who obtained analytical solutions for the velocity and temperature just downstream of a jump in the prescribed plate temperature. In an analysis of the effect of transpiration cooling on free convection, Clarke [9] pointed out that the restriction to small temperature differences can easily be avoided by use of 
a Howarth transformation of the boundary-layer coordinate measuring distance normal to the plate, if the plate has constant temperature. For a semi-infinite plate he obtained inner and outer solutions for large values of a Grashof number based on a reference distance along the plate, and questioned the omission of plume entrainment effects for a finite plate as proposed by Yang [6].

The present work is concerned with understanding the rather complicated local asymptotic flow structure near a discontinuity in one of the plate boundary conditions, as at a leading or trailing edge or at a jump in plate temperature, and points out the various effects which must be considered for the calculation of the second-order heat transfer from an isothermal plate of finite length. Section 2 describes the boundarylayer formulation and solutions for an isothermal plate, and notes the regions where the boundary-layer equations are expected to be in error. In Section 3 it is noted that the full Navier-Stokes equations are required for a small region near the leading (i.e., lower) edge, and the leading-edge contribution to the heat transfer is calculated. Section 4 gives the forms assumed for the inner and outer asymptotic expansions, at large Grashof numbers, near the trailing (i.e., upper) edge, and shows the order of magnitude of the trailing-edge correction to the total heat transfer. In Section 5 a somewhat similar formulation is given for a small region near a discontinuity in plate temperature, and a solution for the local pressure distribution is obtained.

\section{Boundary-layer and wake solutions}

We consider steady, laminar, two-dimensional free-convection flow past a vertical flat plate of length $L$, at uniform temperature $T_{w}$. The temperature and density far from the plate, taken to be constant, are $T_{\infty}$ and $\rho_{\infty}$ respectively. In the Boussinesq approximation the nondimensional conservation equations are

$$
\begin{aligned}
& u_{x}+v_{y}=0 \\
& u u_{x}+v u_{y}=-p_{x}+\theta+\operatorname{Gr}^{-1 / 2}\left(u_{x x}+u_{y y}\right) \\
& u v_{x}+v v_{y}=-p_{y}+\operatorname{Gr}^{-1 / 2}\left(v_{x x}+v_{y y}\right) \\
& u \theta_{x}+v \theta_{y}=\mathrm{Gr}^{-1 / 2} \operatorname{Pr}^{-1}\left(\theta_{x x}+\theta_{y y}\right)
\end{aligned}
$$

Here $x$ and $y$ are rectangular coordinates made nondimensional with $L$; the origin is at the leading edge, with $x$ measured vertically upward along the plate and $y$ measured normal to the plate. The velocity components $u$ and $v$, in the $x$ and $y$ directions respectively, have been made nondimensional with a typical convection velocity $V_{c}$ $=\left\{g\left(T_{w}-T_{\infty}\right) L / T_{\infty}\right\}^{1 / 2}$, where $g$ is the gravitational acceleration. The nondimensional temperature is $\theta=\left(T-T_{\infty}\right) /\left(T_{w}-T_{\infty}\right)$ and $p$ is the pressure rise above the ambient pressure $p_{\infty}$, made nondimensional with $\rho_{\infty} V_{c}^{2}$. The Reynolds, Grashof, and Prandtl numbers are $\operatorname{Re}=\rho_{\infty} V_{c} L / \mu_{\infty}, \mathrm{Gr}=(\mathrm{Re})^{2}$, and $\operatorname{Pr}=\mu_{\infty} c_{p} / k_{\infty}$, where $\mu$ and $k$ are the viscosity coefficient and the thermal conductivity, with the subscript $\infty$ indicating conditions far from the plate, and $c_{p}$ is the specific heat at constant pressure. The approxi- 
mations used in Eqns. (2.1) through (2.4) assume small temperature changes, so that $T_{w}-T_{\infty} \ll T_{\infty}, \mu$ and $k$ are everywhere taken equal to their undisturbed values, and changes in the density $\rho$ are neglected in the continuity equation. We have also assumed that relative changes in $p$ are much smaller than relative changes in $T$, and that viscous dissipation is negligible. The kinetic energy $\frac{1}{2} \rho_{\infty} V_{c}^{2}$ is therefore assumed much smaller than the changes in enthalpy, and so $g L \ll c_{p} T_{\infty}$; corresponding neglected terms are extremely small for typical values of $L$ and $T_{\infty}$. For related problems in rotating systems, however, the body force is centrifugal rather than gravitational, and the accelerations may be much larger than $g$; if the parameter analogous to $g L /\left(c_{p} T_{\infty}\right)$ is not negligible, a second-order correction accounting for viscous dissipation can be worked out [10]. Finally, since the motion is symmetric about $y=0$, we need only consider the half-plane $y>0$. The boundary conditions for Eqns. (2.1) through (2.4) are then

$$
\begin{aligned}
& y=0, \quad 0<x<1: \quad u=v=0, \quad \theta=1 \\
& y=0, \quad-\infty<x<0 \quad \text { and } 1<x<\infty: \quad v=0, \quad u_{y}=\theta_{y}=0 \\
& x^{2}+y^{2} \rightarrow \infty: \quad u, v, \theta, p \rightarrow 0
\end{aligned}
$$

At large values of the Grashof number, heat is conducted away from the plate very slowly, so that the heated fluid remains in a thin boundary layer along the plate and in a thin plume, or wake, above the plate (provided that $\operatorname{Pr~Gr}^{1 / 2}$ is also large). The fluid outside these regions is undisturbed in a first approximation, so that the approximate solutions valid outside the boundary layer and plume are

$$
u=v=\theta=p=0
$$

In the thin layers the velocity and temperature are described by boundary-layer equations:

$$
\begin{aligned}
& u_{x}+v_{y}=0 \\
& u u_{x}+v u_{y}=\theta+\varepsilon^{2} u_{y y} \\
& 0=p_{y} \Rightarrow p=0 \\
& u \theta_{x}+v \theta_{y}=\varepsilon^{2} \operatorname{Pr}^{-1} \theta_{y y}
\end{aligned}
$$

where we have set $\varepsilon^{2} \equiv \mathrm{Gr}^{-1 / 2}$. It was recognized by Clarke [9] that the assumption of small temperature and density changes is unnecessary if the $y$-coordinate in Eqns. (2.9) through (2.12) is interpreted as a new variable defined according to a Howarth transformation. Now we take $\rho \mu /\left(\rho_{\infty} \mu_{\infty}\right)=C$, where the constant $C$ is a representative value, and $\mathrm{Pr}$ is also assumed constant. The required replacements are

$$
\begin{aligned}
& y \rightarrow \int_{0}^{y} \frac{\rho}{\rho_{\infty}} d y \\
& v \rightarrow \frac{\rho}{\rho_{\infty}} v+u \frac{\partial}{\partial x} \int_{0}^{y} \frac{\rho}{\rho_{\infty}} d y \\
& \operatorname{Gr}^{1 / 2} \rightarrow \mathrm{Gr}^{1 / 2} / C
\end{aligned}
$$


Since the boundary-layer equations are parabolic, no signals are sent upstream, and so in the boundary-layer approximation the presence of the trailing edge does not influence the flow along the plate. Thus the first-order solution for $0<x<1$ is the similarity solution for a semi-infinite plate $[3,4]$ :

$$
\begin{aligned}
& u=(4 x)^{1 / 2} f_{0}^{\prime}(\eta) \\
& v=-\varepsilon(4 x)^{-1 / 4}\left(3 f_{0}-\eta f_{0}^{\prime}\right) \\
& \theta=g_{0}(\eta)
\end{aligned}
$$

where

$$
\eta=Y(4 x)^{-1 / 4}, \quad Y=y / \varepsilon, \quad \varepsilon=\mathrm{Re}^{-1 / 2}=\mathrm{Gr}^{-1 / 4}
$$

and $f_{0}, g_{0}$ satisfy the ordinary differential equations

$$
\begin{aligned}
& f_{0}^{\prime \prime \prime}+3 f_{0} f_{0}^{\prime \prime}-2 f_{0}^{\prime 2}+g_{0}=0 \\
& g_{0}^{\prime \prime}+3 \operatorname{Pr} f_{0} g_{0}^{\prime}=0
\end{aligned}
$$

subject to boundary conditions

$$
f_{0}(0)=f_{0}^{\prime}(0)=f_{0}^{\prime}(\infty)=g_{0}(0)-1=g_{0}(\infty)=0
$$

The local nondimensional heat flux from the plate is

$$
-\theta_{y}(x, 0)=-C^{-1 / 2} \mathrm{Gr}^{1 / 4}(4 x)^{-1 / 4} g_{0}^{\prime}(0)
$$

The Nusselt number to be used here is an average value equal to the total heat flux made nondimensional with $k_{w} L\left(T_{w}-T_{\infty}\right)$, where $k_{w}=k\left(T_{w}\right)$ :

$$
\mathrm{Nu}=-\int_{0}^{1} \theta_{y} d x=-\frac{4^{3 / 4}}{3} C^{-1 / 2} \mathrm{Gr}^{1 / 4} g_{0}^{\prime}(0)
$$

Values of $g_{0}^{\prime}(0)$ were tabulated by Ostrach [4] for several values of $\operatorname{Pr}$; Ede [2] has summarized these and other numerical results. Kuiken [11,12] has derived asymptotic expansions for $g_{0}^{\prime}(0)$ for large and small $\mathrm{Pr}$.

In the plume, for $1<x<\infty$, no simple solution exists, but the asymptotic behavior for $x \rightarrow 1$ and $x \rightarrow \infty$ can be determined. For $x \rightarrow \infty$, Yih [13] gave similarity solutions expressing $u x^{-1 / 5}$ and $\theta x^{3 / 5}$ as functions of $Y x^{-2 / 5}$, consistent with the differential equations and boundary conditions and with the requirement that the heat convected across surfaces $x=$ constant be independent of $x$. For $x \rightarrow 1$ a solution analogous to Goldstein's [14] near-wake solution was given by Yang [6]. Near $Y=0$, the first terms in $u(x-1)^{-1 / 3}$ and $\theta-1$ are functions of a similarity variable $Y(x-1)^{-1 / 3}$. In the remainder of the thin plume, for $x \rightarrow 1$ with $Y$ fixed, the leading terms in $u$ and $\theta$ are obtained from the boundary-layer solutions (2.16) and (2.18) evaluated at the trailing edge $x=1$. The acceleration of the fluid for $Y=O\left((x-1)^{1 / 3}\right)$ requires a small inflow toward $Y=0$, and in the main part of the thin layer the resulting displacement effect gives $v=O\left(\varepsilon(x-1)^{-2 / 3}\right)$, singular at $x=1$ and implying a second term in $u$ which is of order $(x-1)^{1 / 3}$. Yang constructed inner and outer series 
solutions proceeding in powers of $(x-1)^{1 / 3}$ with functions of $Y(x-1)^{-1 / 3}$ and of $Y$, respectively, as coefficients. Boundary conditions are satisfied at $Y(x-1)^{-1 / 3}=0$ and as $Y \rightarrow \infty$, and the inner and outer expansions are required to match asymptotically as $Y \rightarrow 0$ and $Y(x-1)^{-1 / 3} \rightarrow \infty$. Yang then completed the plume solution in an approximate way by using an integral method to effect a joining of the solutions for $x \rightarrow 1$ and for $x \rightarrow \infty$.

Outside the boundary layer and plume the effects of viscosity and heat conduction are very small, so that the first approximation to the complex velocity $u-i v$ is an analytic function of $z=x+i y$. For a semi-infinite plate the value of $v$ as $y \rightarrow 0$ for $x>0$ describes the mass entrained in the boundary layer, and is found by letting $\eta \rightarrow \infty$ in Eqn. (2.17) to be $v \sim-3 \varepsilon(4 x)^{-1 / 4} f_{0}(\infty)$. For $x<0$, symmetry requires $v \rightarrow 0$ as $y \rightarrow 0$. The solution for the induced external flow has the form $u-i v \sim$ $\left(C_{R}+i C_{I}\right) z^{-1 / 4}$, where the real constants $C_{R}, \mathrm{C}_{I}$ are found from the boundary values of $v$ for $\arg z \rightarrow 0$ and $\arg z \rightarrow \pi$. One obtains

$$
\begin{aligned}
& u \sim 3 \varepsilon f_{0}(\infty)\left(x^{2}+y^{2}\right)^{-1 / 8} \sin \left\{\frac{1}{4} \tan ^{-1} \frac{y}{x}+\frac{\pi}{4}\right\} \\
& v \sim-3 \varepsilon f_{0}(\infty)\left(x^{2}+y^{2}\right)^{-1 / 8} \cos \left\{\frac{1}{4} \tan ^{-1} \frac{y}{x}+\frac{\pi}{4}\right\}
\end{aligned}
$$

An equivalent result was given by Clarke [9].

Equation (2.25), evaluated as $y \rightarrow 0$ for $x>0$, is used to provide a matching condition as $\eta \rightarrow \infty$ for the second-order term in the boundary layer solution for $u$ for the semi-infinite plate $[5,9]$. The corresponding perturbation in $\theta$ is found to be zero, and so there is no second-order term in the local heat transfer $\theta_{y}$. For a finite plate, Yang and Jerger [5] considered mass entrainment only along the plate, setting $v \sim-3 \varepsilon(4 x)^{-1 / 4} f_{0}(\infty)$ for $0<x<1$ and $v \sim 0$ for $1<x<\infty$, as $y \rightarrow 0$ and $\eta \rightarrow \infty$. Since the corresponding second-order boundary-layer solution does not have a similarity form, the term of order $\varepsilon$ in $\theta$ is not zero, for the finite plate, and a term of order one in the Nusselt number is obtained. However, as Clarke [9] has noted, numerical values for the effect of plume entrainment on this term have not been given. The effect could probably be estimated rather accurately from the results of the integral method used by Yang [6] for an approximate joining of the near- and far-wake solutions.

However, improved accuracy in the local heat transfer over most of the plate is not sufficient for calculation of the correct second-order term in the integrated heat transfer $\mathrm{Nu}$. The similarity solution for a semi-infinite plate is singular at the leading edge and Yang's [6] plume solution is singular at the trailing edge. Thus it should be clear that the boundary-layer equations must be modified near the edges, and that higher-order terms in the integrated Nusselt number can be influenced by changes in the local heat transfer in regions near the edges. In other words, the solution of Yang and Jerger for the second-order Nusselt number may be somewhat inaccurate because of the incomplete calculation of the local heat transfer at points away from the edges, 
and still larger contributions may be missing because the effects of leading and trailing edges have not been included. The leading-edge contribution to the Nusselt number is given explicitly below in Section 3, and the order of magnitude of the trailing-edge correction is shown in Section 4.

\section{The leading-edge region}

For a semi-infinite plate the geometric length $L$ is artificial. We introduce a different characteristic length and characteristic velocity, respectively, as

$$
\begin{aligned}
\varepsilon^{4 / 3} L & =\nu_{\infty}^{2 / 3}(g \Delta)^{-1 / 3} \\
\varepsilon^{2 / 3} V_{c} & =\left(\nu_{\infty} g \Delta\right)^{1 / 3}
\end{aligned}
$$

where we have set $\nu_{\infty}=\mu_{\infty} / \rho_{\infty}$ and $\Delta=\left(T_{w}-T_{\infty}\right) / T_{\infty}$; as before, $\varepsilon=\mathrm{Gr}^{-1 / 4}=$ $\left(V_{c} L / \nu_{\infty}\right)^{-1 / 2}$ and $V_{c}^{2}=g L \Delta$. The new reference values are independent of $L$, and are chosen in such a way that all the parameters disappear from the differential equations and boundary conditions. A set of variables made nondimensional with these values can be related to the variables used previously by

$$
\begin{array}{ll}
x=\varepsilon^{4 / 3} \tilde{x}, & y=\varepsilon^{4 / 3} \tilde{y} \\
u=\varepsilon^{2 / 3} \tilde{u}, & v=\varepsilon^{2 / 3} \tilde{v}, \quad p=\varepsilon^{4 / 3} \tilde{p}, \quad \theta=\tilde{\theta}
\end{array}
$$

The length $L$ disappears in each of these definitions. We could also have obtained the orders of magnitude shown by observing that the similarity solution, Eqns. (2.16) through (2.19), gives $v / u \ll 1$ as required for the validity of the boundary-layer equations only if $x \gg \varepsilon^{4 / 3}, u \gg \varepsilon^{2 / 3}$, etc. In the Boussinesq approximation the differential equations are Eqns. (2.1) through (2.4) with a tilde placed over each variable and with the factors $\varepsilon^{2}$ omitted.

The asymptotic behavior of the solution to these equations in the new variables can be studied for small and large values of the coordinates. If $\tilde{x}^{2}+\tilde{y}^{2} \rightarrow 0$, the largest terms in the energy equation give Laplace's equation for $\tilde{\theta}$, corresponding to steadystate heat conduction. The solution satisfying $\tilde{\theta}(\tilde{x}, 0)=1$ for $\tilde{x}>0$ and $\tilde{\theta}_{\tilde{y}}(\tilde{x}, 0)=0$ for $\tilde{x}<0$ is $\tilde{\theta} \sim 1+A \tilde{r}^{1 / 2} \sin \frac{1}{2} \tilde{\phi}$, where $\tilde{r}^{2}=\tilde{x}^{2}+\tilde{y}^{2}, \tilde{\phi}=\tan ^{-1}(\tilde{y} / \tilde{x})$, and $A$ is a constant which could be determined if the full solution were known. If $\tilde{x} \rightarrow \infty$, the similarity solution in the form given by Eqns. (2.16) through (2.19) is recovered; the definition (2.19) for the similarity variable $\eta$ is equivalent to $\eta=\tilde{y} /(4 \tilde{x})^{1 / 4}$. Improvement of this approximation by the construction of asymptotic expansions for $\mathrm{Gr} \rightarrow \infty$ was considered briefly at the end of Section 2 . For the semi-infinite plate these parameter expansions are more properly written as coordinate expansions for $\tilde{x} \rightarrow \infty$. If second-order terms given by Yang and Jerger [5] and Clarke [9] are retained, we have

$$
\begin{aligned}
& \tilde{u}=(4 \tilde{x})^{1 / 2} f_{0}^{\prime}+(4 \tilde{x})^{-1 / 4} f_{1}^{\prime}+\cdots \\
& \tilde{v}=(4 \tilde{x})^{-1 / 4}\left(-3 f_{0}+\eta f_{0}^{\prime}\right)+(4 \tilde{x})^{-1} \eta f_{1}^{\prime}+\cdots \\
& \tilde{\theta}=g_{0}+o\left(\tilde{x}^{-3 / 4}\right)
\end{aligned}
$$


where $f_{0}$ and $g_{0}$ are the same functions of $\eta=Y(4 x)^{-1 / 4}=\tilde{y}(4 \tilde{x})^{-1 / 4}$ as introduced in Eqns. (2.16) through (2.18) and satisfy Eqns. (2.20) through (2.22); $f_{1}$ satisfies

$$
\begin{aligned}
& f_{1}^{\prime \prime \prime}+3 f_{0} f_{1}^{\prime \prime}-f_{0}^{\prime} f_{1}^{\prime}=0 \\
& f_{1}(0)=f_{1}^{\prime}(0)=0, \quad f_{1}^{\prime}(\infty)=3 f_{0}(\infty)
\end{aligned}
$$

and $\tilde{\theta}$ contains no term of order $\tilde{x}^{-3 / 4}$.

The coordinate expansions in Eqns. (3.3) through (3.5) describe the flow for $\tilde{x} \gg 1$, and the second-order term in the local heat transfer is zero, as also found by use of the parameter expansions for $\mathrm{Gr} \rightarrow \infty$ with $x$ fixed, described in Section 2 . Therefore integration of $\theta_{y}$ along the plate from $x=0$ to $x=1$ appears to give the result that the second term in $\mathrm{Nu}$ is zero. However, the boundary-layer equations are not correct near the leading edge, for $\tilde{x}=O(1)$, and the first-order boundary-layer solution for $\theta_{y}$ has an error of order $\varepsilon^{-4 / 3}$ when $x=O\left(\varepsilon^{4 / 3}\right)$. Thus by integrating this solution along the plate we lose a term in $\mathrm{Nu}$ which is of order one.

Without actually obtaining the details for $x=O\left(\varepsilon^{4 / 3}\right)$, however, we can obtain the integrated heat transfer from the plate up to a location $\varepsilon^{4 / 3} \tilde{x} \equiv x=1$ by using an integrated energy equation. The procedure is analogous to Imai's [15] calculation of the leading-edge contribution to the drag coefficient for a flat plate in a uniform incompressible stream at high Reynolds number. We first combine the energy equation with the continuity equation and rewrite it in the nondimensional integral form

$$
\frac{1}{\operatorname{Pr}} \int_{S} \nabla \tilde{\theta} \cdot \vec{n} d A=\int_{S} \tilde{\theta} \vec{u} \cdot \vec{n} d A
$$

where differentiation and integration are carried out with respect to $\tilde{x}$ and $\tilde{y}$. The control surface $S$ can be taken as a large rectangle with sides, say, $x= \pm 1, y=0$, and $y=1$. Along $y=0$, the convection term is zero because $\tilde{v}=0$, and the heat conduction term contributes only for $\tilde{x}>0$ because $\tilde{\theta}_{\tilde{y}}=0$ for $\tilde{x}<0$. At $x=1$, within the boundary layer the convection term contributes quantities which are $O\left(\tilde{x}^{3 / 4}\right)$ and $O(1)$, whereas the conduction term is $O\left(\tilde{x}^{-3 / 4}\right)$. Finally, outside the boundary layer the velocity is of order $\varepsilon V_{c}$, so that $c_{p}\left(T-T_{\infty}\right)=O\left(\varepsilon^{2} V_{c}^{2}\right)$ and $\tilde{\theta}$ is extremely small. Thus the largest terms in the integrated energy equation are the terms representing heat conduction from the plate and convection of heat within the boundary layer:

$$
\begin{array}{r}
-\int_{0}^{\tilde{x}} \tilde{\theta}_{\tilde{y}} d \tilde{x} \sim \operatorname{Pr} \int_{0}^{\infty}\left\{g_{0}(\eta)+o\left(\tilde{x}^{-3 / 4}\right)\right\}\left\{(4 \tilde{x})^{1 / 2} f_{0}^{\prime}(\eta)+(4 \tilde{x})^{-1 / 4} f_{1}^{\prime}(\eta)\right. \\
+\cdots\}(4 \tilde{x})^{1 / 4} d \eta
\end{array}
$$

On the right-hand side we integrate the largest term by parts and replace $g_{0}^{\prime} f_{0}$ by $-(3 \mathrm{Pr})^{-1} g_{0}^{\prime \prime}$. If the temperature changes are not small, the transformation given by Eqns. (2.13) through (2.15) can be used, and the Nusselt number for the portion of the plate up to a distance $L$ from the leading edge is then found by setting $\tilde{x}=\varepsilon^{-4 / 3}$ :

$$
\mathrm{Nu}=-\frac{4^{3 / 4}}{3} C^{-1 / 2} \mathrm{Gr}^{1 / 4} g_{0}^{\prime}(0)+\operatorname{Pr} \int_{0}^{\infty} g_{0}(\eta) f_{1}^{\prime}(\eta) d \eta
$$


As noted above, for a semi-infinite plate the second-order term in the local heat transfer is correctly given as zero by the perturbation procedure which leads to Eqns. (3.3) through (3.5). Equation (3.10) accounts for the leading-edge contribution to the integrated heat transfer in terms of the function $f_{1}^{\prime}$, where $f_{1}^{\prime}$ is obtained by perturbing about the boundary-layer solution at a distance from the leading edge which is large in comparison with $\nu_{\infty}^{2 / 3}(g \Delta)^{-1 / 3}$. The result, of course, refers to the idealized case of a plate having zero thickness, which would be approximated satisfactorily if the leadingedge shape were that of a very slender wedge. For a plate of finite length, the secondorder local heat transfer would not be zero. The velocity perturbation given by $f_{1}^{\prime}$ in Eqn. (3.10) would no longer have a similarity form, and the right-hand side of Eqn. (3.8) would give an additional term in the integrated heat transfer shown in Eqn. (3.10), equal to the integral of $f_{0}^{\prime}(\eta)$ multiplied by the second term in $\theta$. The effect of the leading edge, however, would not be changed, since each of these additional effects in Eqn. (3.10) would be proportional to a positive power of the distance from the leading edge, and so would decrease toward zero as this distance is decreased.

For $\operatorname{Pr}=0.72$ and $\operatorname{Pr}=10.0$ the values of the correction given in Eqn. (3.10) are found by numerical integration to be 0.623 and 0.457 , respectively. For the same values of Pr, the corrections obtained by Yang and Jerger [5] to account for finite plate length are, respectively, -0.312 and -0.216 . The latter results represent a decrease in heat transfer from the plate, probably somewhat overestimated because the effect of entrainment into the plume was neglected. The leading-edge correction, on the other hand, gives an increase in $\mathrm{Nu}$, and the combined results likewise show an increase. For example, if $\mathrm{Gr}=10^{4}$ the net increase is about 6.5 per cent for $\mathrm{Pr}=0.72$ and about 2.2 per cent if $\operatorname{Pr}=10.0$, and the corrections become increasingly important as $\mathrm{Gr}$ decreases. The remaining second-order correction, for the effect of the trailing edge, is discussed in the next section.

\section{The trailing-edge region}

In this section we will obtain the equations for a first approximation to the flow near the trailing edge in the limit as $\varepsilon=\mathrm{Gr}^{-1 / 4} \rightarrow 0$. For convenience we redefine the length $L$ as four times the plate length, so that at the trailing edge $4 x=1$ and $\eta \equiv Y(4 x)^{-1 / 4}=Y$. We also define a coordinate $x_{1}$ measured from the trailing edge by $x_{1}=x-\frac{1}{4}$.

The asymptotic flow structure near the trailing edge is in some ways similar to that for a flat plate placed in a uniform incompressible stream. The discontinuous change in boundary conditions at the edge implies large derivatives with respect to $x$ in some small region. The accelerations are much larger than their values upstream, but the velocity changes are small, so that an approximate velocity profile is found from Eqn. (2.16) evaluated at the trailing edge, and the viscous forces are changed only slightly from their values just upstream. One therefore anticipates a description of the perturbations in the boundary-layer flow in terms of inviscid-flow equations. Since 
solutions to these equations will not satisfy all the boundary conditions at $Y=0$, one further expects that the flow in a viscous sublayer near $Y=0$ must still be described by boundary-layer equations, but with a nonzero pressure gradient. For the trailing-edge problem considered by Stewartson [16] and Messiter [17] the pressure gradient was necessary because of interaction of the boundary layer with the external flow. In the present case there is no external flow in the limit as $\varepsilon \rightarrow 0$, and so the pressure outside the boundary layer is very nearly constant. In order that a streamwise pressure gradient $p_{x}$ may appear in the sublayer equations, the transverse pressure gradient $p_{y}$ must be nonzero in the first approximation for the main part of the boundary layer.

These ideas are expressed in a formal way by the introduction of assumed asymptotic forms for the flow variables in the sublayer. Six small parameters are to be determined: the characteristic lengths in the $x$ and $y$ directions and the quantities which measure the sizes of the dependent variables $u, v, p, \theta-1$ in terms of $\varepsilon$. As in the case of a flat plate placed in a uniform stream, the differential equations provide three of the six conditions needed. In the continuity equation the terms $u_{x}$ and $v_{y}$ are of the same order; in the sublayer momentum equation the viscous and inertia forces are of the same order; and $p$ and $u^{2}$ are of the same order in the sublayer. Two more conditions follow from the requirements that upstream of the trailing-edge region the sublayer solutions for $u$ and $\theta-1$ match asymptotically with the undisturbed velocity and temperature profiles, found by evaluating Eqns. (2.16) and (2.18) for $x=\frac{1}{4}$ and $Y \rightarrow 0$. If the sublayer is assumed to have thickness of order $\varepsilon^{\alpha}$, we have $Y=$ $O\left(\varepsilon^{\alpha}\right)$ and $y=O\left(\varepsilon^{1+\alpha}\right)$ there, where $\alpha$ is a number to be determined. It then follows from the preceding considerations that the length of the region of interest is of order $\varepsilon^{3 \alpha}$, so that $x_{1}=O\left(\varepsilon^{3 \alpha}\right)$. Thus we take the limit $\varepsilon \rightarrow 0$ with $\varepsilon^{-3 \alpha} x_{1}$ and $\varepsilon^{-\alpha} Y$ held fixed. It also follows that $u=O\left(\varepsilon^{\alpha}\right), v=O\left(\varepsilon^{1-\alpha}\right), p=O\left(\varepsilon^{2 \alpha}\right)$, and $\theta-1=O\left(\varepsilon^{\alpha}\right)$ in the sublayer.

Up to this point the formulation is identical to that for a trailing edge in a uniform external stream, without the condition which represents the interaction effect. We must next obtain an additional condition relating the perturbation in pressure and some function derived from one or more of the other variables. The first terms in the sublayer solutions for velocity and temperature, evaluated for $\varepsilon^{-\alpha} Y \rightarrow \infty$, must match with the undisturbed profiles as $Y \rightarrow 0$, and so $u \sim Y f_{0}^{\prime \prime}(0)$ and $\theta-1 \sim Y g_{0}^{\prime}(0)$. We will assume that the second terms are of order $\varepsilon^{\alpha}$. Using the continuity equation and assuming also that the first term in $p$ is independent of $\varepsilon^{-\alpha} Y$ in the thin sublayer, we have $v=O\left(\varepsilon^{1-2 \alpha} Y\right)$ and $p=O\left(\varepsilon^{2 \alpha}\right)$ as $\varepsilon^{-\alpha} Y \rightarrow \infty$. In the main part of the boundary layer, for proper matching, the perturbations in $u$ and $\theta$ and the solutions for $v$ and $p$ must then be of order $\varepsilon^{\alpha}, \varepsilon^{\alpha}, \varepsilon^{1-2 \alpha}$, and $\varepsilon^{2 \alpha}$ respectively. If the terms $p_{y}$ and $u v_{x}$ in the $y$-momentum equation are to be of the same order for $Y=O(1)$ and $x_{1}=O\left(\varepsilon^{3 \alpha}\right)$, it follows that $\alpha=2 / 7$.

With this result we now have all the information needed for determining the forms to be assumed for the asymptotic expansions describing the sublayer and the main 
part of the boundary layer. The trailing-edge region is defined by $x_{1}=O\left(\varepsilon^{6 / 7}\right)$ and so extends a vertical distance slightly larger, in order of magnitude, than the boundarylayer thickness. In the sublayer, $Y=O\left(\varepsilon^{2 / 7}\right)$. These results permit certain self-consistent approximations to be made in the equations for the largest terms in the expansions, and it will be shown later that the orders of magnitude assumed here are also consistent with Yang's [6] downstream solution obtained for $\varepsilon \rightarrow 0, x_{1} \rightarrow 0$. The approximate problem formulation will indicate that there is a significant local trailingedge contribution to the second-order heat transfer from the plate. Clearly, however, fractional powers of $\varepsilon$ will not really be very small numbers for realistic values of $\mathrm{Gr}$, and an improved approximation may be necessary if good numerical accuracy is required.

The flow in the main part of the boundary layer is described by equations obtained as $\varepsilon \rightarrow 0$ with coordinates $\xi$ and $Y$ held fixed, where

$$
\xi=\varepsilon^{-6 / 7} x_{1}=\varepsilon^{-6 / 7}\left(x-\frac{1}{4}\right), \quad Y=\varepsilon^{-1} y
$$

The following expansions are assumed:

$$
\begin{aligned}
u & \sim f_{0}^{\prime}(Y)+\varepsilon^{2 / 7} U_{1}(\xi, Y)+\cdots \\
v & \sim \varepsilon^{3 / 7} V_{1}(\xi, Y)+\cdots \\
p & \sim \varepsilon^{4 / 7} P_{1}(\xi, Y)+\cdots \\
\theta & \sim g_{0}(y)+\varepsilon^{2 / 7} \Theta_{1}(\xi, Y)+\cdots
\end{aligned}
$$

The differential equations to be satisfied are

$$
\begin{aligned}
& U_{1 \xi}+V_{1 Y}=0 \\
& f_{0}^{\prime}(Y) U_{1 \xi}+f_{0}^{\prime \prime}(Y) V_{1}=0 \\
& f_{0}^{\prime}(Y) V_{1 \xi}=-P_{1 Y} \\
& f_{0}^{\prime}(Y) \Theta_{1 \xi}+g_{0}^{\prime}(Y) V_{1}=0
\end{aligned}
$$

Eliminating $U_{1}$ from Eqns. (4.6) and (4.7), and integrating, we obtain

$$
V_{1}(\xi, Y)=-f_{0}^{\prime}(Y) G^{\prime}(\xi)
$$

where $G(\xi)$ is a function to be determined, and it is expected that $G^{\prime}(\xi) \rightarrow 0$ as $\xi \rightarrow-\infty$. Eqn. (4.8) then gives, after integration,

$$
P_{1}(\xi, Y)=-G^{\prime \prime}(\xi) \int_{Y}^{\infty} f_{0}^{\prime 2}(Y) d Y
$$

where we have set $P_{1}(\xi, \infty)=0$. Substituting Eqn. (4.10) in Eqns. (4.7) and (4.9) and integrating, we also have

$$
\begin{aligned}
& U_{1}(\xi, Y)=f_{0}^{\prime \prime}(Y) G(\xi) \\
& \Theta_{1}(\xi, Y)=g_{0}^{\prime}(Y) G(\xi)
\end{aligned}
$$

We require $U_{1}, \Theta_{1} \rightarrow 0$ as $\xi \rightarrow-\infty$, and so the integration constant in $G(\xi)$ is chosen such that $G(\xi) \rightarrow 0$ as $\xi \rightarrow-\infty$. Temperature changes have been assumed small in 
Eqns. (4.1) through (4.13). If ( $\left.T_{w}-T_{\infty}\right) / T_{\infty}$ is not small, the density should appear as an additional variable. The equation of state gives $\rho / \rho_{\infty}=T_{\infty} / T+O\left(\varepsilon^{4 / 7}\right)$, and so it is found that

$$
\frac{\rho}{\rho_{\infty}}=\frac{1}{1+g_{0}(Y) \Delta}\left\{1-\frac{\varepsilon^{2 / 7}}{1+g_{0}(Y) \Delta} \Theta_{1}(\xi, Y)+\cdots\right\}
$$

where we have set $\Delta \equiv\left(T_{w}-T_{\infty}\right) / T_{\infty}$, so that $T / T_{\infty} \equiv 1+\Delta \theta$. If the derivation is repeated for variable density, the results given by Eqns. (4.10) through (4.13) remain correct provided that $Y$ and $V_{1}$ are interpreted as transformed variables obtained using the first approximations to Eqns. (2.13) and (2.14).

The equations describing the flow in the sublayer are obtained by taking a limit as $\varepsilon \rightarrow 0$ with coordinates $\xi$ and $\zeta$ held fixed:

$$
\xi=\varepsilon^{-6 / 7} x_{1}=\varepsilon^{-6 / 7}\left(x-\frac{1}{4}\right), \quad \zeta=\varepsilon^{-2 / 7} Y=\varepsilon^{-9 / 7} y
$$

The following expansions are assumed:

$$
\begin{aligned}
u(x, y) & \sim \varepsilon^{2 / 7} u_{1}(\xi, \zeta)+\cdots \\
v(x, y) & \sim \varepsilon^{5 / 7} v_{1}(\xi, \zeta)+\cdots \\
p(x, y) & \sim \varepsilon^{4 / 7} p_{1}(\xi)+\cdots \\
\theta(x, y) & \sim 1+\varepsilon^{2 / 7} \theta_{1}(\xi, \zeta)+\cdots
\end{aligned}
$$

The corresponding differential equations are

$$
\begin{aligned}
& u_{1 \xi}+v_{1 \zeta}=0 \\
& u_{1} u_{1 \xi}+v_{1} u_{1 \zeta}=-p_{1}^{\prime}+u_{1 \zeta \zeta} \\
& u_{1} \theta_{1 \xi}+v_{1} \theta_{1 \zeta}=\frac{1}{\operatorname{Pr}} \theta_{1 \zeta \zeta}
\end{aligned}
$$

The boundary conditions at $Y=0$ are

$$
\begin{aligned}
& u_{1}(\xi, 0)=v_{1}(\xi, 0)=\theta_{1}(\xi, 0)=0, \quad \xi<0 \\
& u_{1 \xi}(\xi, 0)=v_{1}(\xi, 0)=\theta_{1 \xi}(\xi, 0)=0, \quad \xi>0
\end{aligned}
$$

For $\xi \rightarrow-\infty$, matching with the initial profiles for $Y \rightarrow 0$ gives

$$
u_{1}(\xi, \zeta) \sim f_{0}^{\prime \prime}(0) \zeta, \quad \theta_{1}(\xi, \zeta) \sim g_{0}^{\prime}(0) \zeta
$$

Matching the velocity, temperature and pressure for $\zeta \rightarrow \infty$ and $Y \rightarrow 0$ gives

$$
\begin{aligned}
& u_{1}(\xi, \zeta) \sim f_{0}^{\prime \prime}(0)\{\zeta+G(\xi)\}, \quad \theta_{1}(\xi, \zeta) \sim g_{0}^{\prime}(0)\{\zeta+G(\xi)\} \\
& p_{1}(\xi)=P_{1}(\xi, 0)=-G^{\prime \prime}(\xi) \int_{0}^{\infty} f_{0}^{\prime 2}(Y) d Y
\end{aligned}
$$

If the temperature difference $\Delta=\left(T_{w}-T_{\infty}\right) / T_{\infty}$ is not small, the replacements proposed in Eqns. (2.13) through (2.15) give $\varepsilon^{2} \rightarrow \varepsilon^{2} / C$ and, since $Y \ll 1, y \rightarrow \rho_{w} y / \rho_{\infty}$ and $v \rightarrow \rho_{w} v / \rho_{\infty}$. 
Equations (4.20) through (4.26) might be considered as describing a type of boundary-layer interaction problem. The pressure gradient $\varepsilon^{-2 / 7} p_{1}^{\prime}(\xi)$ is not specified in advance, but is related to the streamline curvature $-\varepsilon^{-3 / 7} G^{\prime \prime}(\xi)$ by Eqn. (4.26), and is interpreted in the following way. Near $Y=0$ the fluid accelerates because the restraining force on the plate is removed at $\xi=0$. The corresponding displacement effect requires a small inward mass flow toward $Y=0$, and the fluid acceleration corresponding to the strong streamline curvature implies a pressure gradient along the direction normal to the plate. Since the pressure outside the boundary layer is very nearly constant, it follows that there must also be a pressure gradient along $Y=0$, which contributes to the net force acting on the fluid in the sublayer. As $\xi$ increases, the functions $-G^{\prime \prime}(\xi)$ and $p_{1}(\xi)$ initially decrease from zero and become negative, but later increase through zero to positive values, before eventually returning to zero. The relationship between $p_{1}$ and $G^{\prime \prime}$ is a local one, in contrast with the integral relation for a plate in a uniform external stream. As in the latter case, however, it can be argued that $p^{\prime}(\xi)=O\left(\xi^{-1 / 3}\right)$ as $\xi \rightarrow 0$ [17], and that this singular behaviour may have to be taken into account in a numerical solution. For $\xi \rightarrow-\infty$, the forms of solution can be found by the procedure used by Stewartson and Williams [18] in studying separation of a supersonic laminar boundary layer.

The assumed forms of solution given by Eqns. (4.1) through (4.5) and (4.15) through (4.19) can be shown to be consistent with the wake solution given by Yang [6]. In his outer solution for $x_{1} \rightarrow 0$ with $Y$ fixed, it is found that $v=O\left(\varepsilon x_{1}^{-2 / 3}\right)$ and so the $y$-momentum equation $p_{y} \sim-u v_{x}$ gives $p=O\left(\varepsilon^{2} x_{1}^{-5 / 3}\right)$ as $Y \rightarrow 0$. The first term in Yang's solution for small $Y$ is just Goldstein's similarity solution for the merging of two uniform shear flows, so that $u^{2}=O\left(x_{1}^{2 / 3}\right)$. This solution is derived for values of $x_{1}$ which are small but not so small that the pressure gradient is important. For $Y \rightarrow 0, p$ is no longer small compared with $u^{2}$ if $x_{1}=O\left(\varepsilon^{6 / 7}\right)$. Thus Yang's solution describes the flow for $\varepsilon^{6 / 7} \ll\left|x_{1}\right| \ll 1$. If we were to set $x_{1}=O\left(\varepsilon^{6 / 7}\right)$ in this solution, it would follow for $Y=O\left(\varepsilon^{2 / 7}\right)$ that $u=O\left(\varepsilon^{2 / 7}\right), v=O\left(\varepsilon^{5 / 7}\right), p=O\left(\varepsilon^{4 / 7}\right)$ and $\theta-1=O\left(\varepsilon^{2 / 7}\right)$, as already given in Eqns. (4.16) through (4.19). In other words, the formulation proposed above could also have been deduced by observing that the wake solution is no longer valid if $x_{1}=O\left(\varepsilon^{6 / 7}\right)$ and then noting the orders of magnitude required for matching as $x \rightarrow 0$ and $\varepsilon^{-6 / 7} x_{1} \rightarrow \infty$. One can also anticipate that the forms of the functions in Eqns. (4.16) through (4.19) as $\xi \rightarrow \infty$ should be $u_{1}=$ $O\left(\xi^{1 / 3}\right), v_{1}=O\left(\xi^{-1 / 3}\right), p_{1}=O\left(\xi^{-5 / 3}\right)$, and $\theta_{1}=O\left(\xi^{1 / 3}\right)$.

As noted in Section 3, the leading term in the Nusselt number $\mathrm{Nu}$ for $\varepsilon \equiv$ $\mathrm{Gr}^{-1 / 4} \rightarrow 0$ is $O\left(\varepsilon^{-1}\right)$, the correction for finite plate length is $O(1)$ and negative, and the leading-edge correction is $O(1)$ and positive. Since the fluid accelerates just ahead of the trailing edge, conduction of heat away from the plate is enhanced here, and the trailing-edge region therefore is expected to give another positive contribution to $\mathrm{Nu}$. Since $\theta_{y}(x, 0)$ differs from its undisturbed value by a factor of order $\varepsilon^{-1}$ over a distance of order $\varepsilon^{6 / 7}$, this correction is $O\left(\varepsilon^{-1 / 7}\right)$. Thus for $\varepsilon \ll 1$ the trailing-edge term is slightly larger in order of magnitude than the 'second-order' contributions over the 
remainder of the plate, and clearly must be considered in any attempt at estimating the largest correction terms in $\mathrm{Nu}$.

A numerical solution to Eqns. (4.20) through (4.26) would be sufficient to give a good qualitative description of the trailing-edge flow and an estimate of the local contribution to the heat transfer. For improved accuracy it would be necessary first of all to retain, for $Y=O(1)$, the pressure gradient $p_{x}$ and the buoyancy force in the undisturbed form $\theta \sim g_{0}(Y)$. These changes would also influence the sublayer through the matching conditions. The viscous shear stress $u_{Y Y}$ for $Y=O(1)$ would enter in a second correction, but the longitudinal stress $\varepsilon^{2} u_{x x}$ appears to be considerably smaller. For a complete analysis, it might also be necessary to consider the slightly smaller region where $x_{1}=O(\varepsilon)$. If $\theta_{y}$ should require still another correction of order $\varepsilon^{-1}$, in a region having length $O(\varepsilon)$, the second term in the trailing-edge correction to $\mathrm{Nu}$ would be $O(1)$. The full Navier-Stokes equations would, however, be required only in a still smaller region where $x_{1}, y=O\left(\varepsilon^{3 / 2}\right) ; u, v=O\left(\varepsilon^{1 / 2}\right) ; p_{x}, p_{y}=O\left(\varepsilon^{-1 / 2}\right)$; $\theta-1=O\left(\varepsilon^{1 / 2}\right)$; and the local contribution to the heat transfer is $O\left(\varepsilon^{1 / 2}\right)$, smaller than the terms considered here.

The same type of edge effect also occurs for a circular disc rotating about its axis of symmetry in fluid otherwise at rest. For small values of the Ekman number $E=$ $\nu /\left(\Omega R^{2}\right)$, where $\nu=$ kinematic viscosity, $\Omega=$ angular velocity of the disc, and $R=$ disc radius, there is a circumferential flow in thin boundary layers along the upper and lower surfaces, and the resulting centripetal acceleration also requires a radial flow. The radial component of the momentum equation has the same form as the momentum equation (2.10), with additional terms in the convective derivative because the flow is axisymmetric and with the buoyancy force replaced by a centrifugal force. Smith [19] has considered this problem, but has concluded that the changes in pressure would be important only within a radial distance of order $E^{1 / 2} R$ from the edge. In his formulation the pressure changes are of order $E^{1 / 6} p_{\infty}$ in the main part of the boundary layer, but are smaller both in a sublayer having thickness of order $E^{2 / 3} R$ and in the flow outside the boundary layer. But $p_{y} \sim u v_{x}$, in our notation, where $v_{x} / u$ is approximately the streamline curvature. Thus Smith's formulation would imply that the integral of the largest term in $u v_{x}$ across the boundary layer would be zero for all values of $x_{1}$ such that $x_{1}=O\left(E^{1 / 2}\right)$, a result which seems unlikely. If instead the arguments used here were again applied, the local flow structure would be found to have exactly the same form as in the present case, and modification of the boundarylayer equations to account for pressure gradients would be necessary in the somewhat larger region where $x_{1}=O\left(E^{3 / 7} R\right)$.

\section{The flow near a discontinuity in plate temperature}

In the preceding section, the trailing-edge flow was described in terms of the pressure perturbation induced by a displacement effect, which is present because of a discontinuous change in boundary condition. This description differs from the asymptotic 
description of boundary-layer interaction with an external flow, because for the free-convection case the external-flow velocity is zero in the limit as $\varepsilon \rightarrow 0$. A freeconvection 'interaction' of the same general type occurs near a jump discontinuity in the prescribed plate temperature. The flow near the temperature jump has a number of features in common with the trailing-edge flow. Since, however, the boundary conditions for the velocity are now continuous, it is found that the sublayer equations are linear, and an analytical solution for the local pressure distribution can be obtained. The derivation is outlined below and in the Appendix. The existence of an explicit flow description near a temperature jump appears to support the assumptions made concerning the asymptotic form of the solution in Section 4 as well as in this section.

We will locate the origin $x=0$ at the temperature jump, with $T_{w}=T_{w}^{(u)}$ upstream, for $x<0$, and $T_{w}=T_{w}^{(d)}$ downstream, for $x>0$. For convenience we now define $L$ as equal to four times the distance from the leading edge to the jump. We also define

$$
\lambda=\frac{T_{w}^{(u)}-T_{\infty}}{T_{w}^{(d)}-T_{\infty}}
$$

where $0<\lambda<1$ if $T_{w}^{(d)}>T_{w}^{(u)}$ and $1<\lambda<\infty$ if $T_{w}^{(d)}<T_{w}^{(u)}$. The nondimensional parameters and variables will all be defined in terms of $T_{w}^{(d)}$. In particular, $\varepsilon$ is now defined as $\lambda^{1 / 4}$ times the $\varepsilon$ defined for $x<0$; that is, now

$$
\varepsilon=\left(\frac{\mu_{\infty}}{\rho_{\infty} L}\right)^{1 / 2}\left(\frac{T_{\infty}}{g\left(T_{w}^{(d)}-T_{\infty}\right) L}\right)^{1 / 4}
$$

The velocity and temperature profiles just upstream of the jump are given by $u \sim$ $\lambda^{1 / 2} f_{0}^{\prime}\left(\lambda^{1 / 4} Y\right)$ and $\theta \sim \lambda g_{0}\left(\lambda^{1 / 4} Y\right)$.

A solution analogous to Goldstein's [14] has been given by Kelleher [8] for the flow at small positive values of $x$, in terms of inner and outer coordinate expansions. The solution shows $v \rightarrow \infty$ as $x \rightarrow 0$ and so there is a small region about $x=0$ where the formulation is no longer valid. As for the trailing-edge flow, we anticipate that in this region dissipative effects are important primarily in a thin sublayer, and orders of magnitude are determined from the following considerations: $u_{x}$ and $v_{y}$ are of the same order; viscous, pressure, and inertia forces are of the same order; upstream of the jump the velocity and temperature match asymptotically with the undisturbed profiles; and the pressure variation enters because the transverse pressure gradient is important for $Y=O(1)$. These conditions are sufficient to give $x=O\left(\varepsilon^{6 / 7}\right)$ and $Y=O\left(\varepsilon^{2 / 7}\right)$, as before. Since, however, one boundary condition remains $u(x, 0)=$ 0 , the solution for the largest term in $u$ is simply $u \sim \lambda^{3 / 4} f_{0}^{\prime \prime}(0) Y$. The change in buoyancy force contributes to the second term in $u$, and it follows from the $x$-momentum equation that the perturbations in $u$ and $p$ are of order $\varepsilon^{4 / 7}$ and $\varepsilon^{6 / 7}$, respectively.

The dependence on $\lambda$ and on the upstream velocity profile can be expressed through constants $\alpha$ and $\beta$ defined by

$$
\alpha=\lambda^{3 / 4} f_{0}^{\prime \prime}(0), \quad \beta=\frac{1}{f_{0}^{\prime \prime}(0)} \int_{0}^{\infty} f_{0}^{\prime 2}\left(\lambda^{1 / 4} Y\right) d\left(\lambda^{1 / 4} Y\right)
$$


The formulation given by Eqns. (4.15) through (4.26) can now be modified as follows:

$$
\begin{aligned}
\xi & =\varepsilon^{-6 / 7} \alpha^{2 / 7} \beta^{-3 / 7} x, \quad \zeta=\varepsilon^{-2 / 7} \alpha^{3 / 7} \beta^{-1 / 7} Y=\varepsilon^{-9 / 7} \alpha^{3 / 7} \beta^{-1 / 7} y \\
u & \sim \varepsilon^{2 / 7} \alpha^{4 / 7} \beta^{1 / 7} \zeta-\varepsilon^{4 / 7} \alpha^{-6 / 7} \beta^{2 / 7} \frac{\lambda}{2} \zeta^{2}+\varepsilon^{4 / 7}(1-\lambda) \alpha^{-6 / 7} \beta^{2 / 7} u_{1}(\xi, \zeta)+\cdots \\
v & \sim \varepsilon(1-\lambda) \alpha^{-1} v_{1}(\xi, \zeta)+\cdots \\
p & \sim \varepsilon^{6 / 7}(1-\lambda) \alpha^{-2 / 7} \beta^{3 / 7} p_{1}(\xi)+\cdots \\
\theta & \sim \lambda+(1-\lambda) \theta_{1}(\xi, \zeta)+\cdots
\end{aligned}
$$

where

$$
\begin{aligned}
& u_{1 \xi}+v_{1 \zeta}=0 \\
& \zeta u_{1 \xi}+v_{1}-u_{1 \zeta \zeta}=-p_{1}^{\prime}+\theta_{1} \\
& \zeta \theta_{1 \xi}=\frac{1}{\operatorname{Pr}} \theta_{1 \zeta \zeta} \\
& \zeta=0, \quad \xi<0: \quad u_{1}=v_{1}=0, \quad \theta_{1}=0 \\
& \zeta=0, \quad \xi>0: \quad u_{1}=v_{1}=0, \quad \theta_{1}=1 \\
& \xi \rightarrow-\infty: \quad u_{1} \sim 0, \quad \theta_{1} \sim 0 \\
& \zeta \rightarrow \infty: \quad u_{1} \sim G(\xi), \quad \theta_{1} \sim 0 \\
& p_{1}(\xi)=-G^{\prime \prime}(\xi)
\end{aligned}
$$

As in Eqn. (4.10), $G^{\prime}(\xi)$ can be interpreted in terms of the streamline slope, since $v / u \sim-\varepsilon^{5 / 7}(1-\lambda) \alpha^{-11 / 7} \beta^{-1 / 7} G^{\prime}(\xi)$ for $Y=O(1)$.

The solution for $\theta_{1}$ remains the same as Kelleher's [8] downstream solution for the change in temperature. For $\xi>0$,

$$
\theta_{1}=\frac{3^{1 / 3}}{\Gamma(1 / 3)} \int_{\zeta(\operatorname{Pr} / \xi)^{1 / 3}}^{\infty} e^{-t^{3 / 9}} d t
$$

For $\xi<0$, however, $\theta_{1}=0$ and so for $x=O\left(\varepsilon^{6 / 7}\right)$ the jump in wall temperature has no upstream influence. We should expect that the temperature ahead of the jump is influenced only at a small distance such that $\theta_{x x}$ is no longer negligible in comparison with $\theta_{y y}$; as in Section 4, $\theta_{x x}$ must be retained when $x=O\left(\varepsilon^{3 / 2}\right), y=O\left(\varepsilon^{3 / 2}\right)$.

Although the change in boundary condition influences $\theta_{1}$ only for $\xi>0$, the perturbations in velocity and pressure are affected both for $\xi>0$ and for $\xi<0$. A solution for $u_{1}(\xi, \zeta)$ in terms of $p_{1}(\xi)$ can be found from Eqns. (5.9) and (5.10), subject to the boundary conditions given in Eqns. (5.12) and (5.13). This sublayer solution must have the form given by Eqn. (5.14) as $\zeta \rightarrow \infty$, and so provides a relation between $G(\xi)$ and $p_{1}(\xi)$, which can be combined with Eqn. (5.15) to give an equation involving only $p_{1}(\xi)$. An outline of this derivation is given in the Appendix, and the solution for $p_{1}(\xi)$ is obtained. For $\xi<0$

$$
p_{1}(\xi)=-\frac{3 B}{7 k A} e^{k \xi}
$$


and for $\xi>0$

$$
\begin{aligned}
p_{1}(\xi)= & \frac{6}{7} \frac{B}{A} e^{-\xi \sin 5 \pi / 14} \sin \left(\xi \cos \frac{5 \pi}{14}-\frac{5 \pi}{14}\right) \\
& +\frac{\sqrt{ } 3}{2 \pi} \frac{B}{A} k^{7 / 3} \int_{0}^{\infty} \frac{t^{1 / 3} e^{-\xi t} d t}{t^{14 / 3}-k^{7 / 3} t^{7 / 3}+k^{14 / 3}}
\end{aligned}
$$

where $A=3^{-2 / 3} \Gamma\left(\frac{1}{3}\right) / \Gamma\left(\frac{2}{3}\right), k^{7 / 3}=3^{2 / 3} / \Gamma\left(\frac{1}{3}\right)$, and $B$ is a positive constant which depends on $\operatorname{Pr}$ and can be obtained in the manner described in the Appendix. The pressure perturbation $p_{1}(\xi)$ is continuous at $\xi=0$, but the derivative has a jump given by $p_{1}^{\prime}(+0)-p_{1}^{\prime}(-0)=B / A$, as can be shown using either Eqn. (5.18) or Eqn. (A.5). As $\xi \rightarrow \infty, p_{1} \sim 3^{-1 / 2}(2 \pi)^{-1} B \Gamma\left(\frac{1}{3}\right) \xi^{-4 / 3} / \Gamma\left(\frac{2}{3}\right)$ and so $p=O\left(\varepsilon^{2} x^{-4 / 3}\right)$. From Kelleher's (1971) solutions for $x \rightarrow 0$, using his function $\Phi_{2}$, one can show that the integral of the streamline curvature across the boundary layer is also $O\left(\varepsilon^{2} x^{-4 / 3}\right)$, in agreement with the result for $p_{1}$ as $\xi \rightarrow \infty$. Since $B>0$, it is seen that $p_{1}(\xi)$ is negative for $\xi<0$, but later becomes positive before returning to zero as $\xi \rightarrow \infty$.

\section{Conclusions}

For the vertical flat plate in free convection, the existence of a similarity solution permits easy calculation of the first approximation to the heat transfer at large Grashof number. However, in spite of the very simple geometry, it is extremely difficult to obtain an adequate correction which extends the range of usefulness of this result to lower values of the Grashof number. It has been attempted here to describe the asymptotic structure of the regions where the boundary-layer equations are no longer valid, and thereby to identify effects which must be accounted for in a second-order calculation and which heretofore have not been recognized. In particular, (1) the leadingedge contribution is obtained explicitly; (2) the trailing-edge effect is shown to be predominantly the effect of a kind of boundary-layer interaction, involving pressure forces but not the longitudinal viscous stresses; and (3) the same kind of interaction is shown to occur near a jump in the plate temperature, by means of an explicit solution for the local pressure distribution.

One of the authors (A.L.) was on leave at The University of Michigan while this study was carried out. This work was supported in part by the U.S. Army Research Office under Contract DAHC $0468 \mathrm{C} 0033$.

\section{Appendix}

The momentum equation (5.10) is linear, and the solution for $u_{1}$ can be written as the sum of solutions $u_{1}^{(\theta)}$ and $u_{1}^{(p)}$ corresponding to the change in buoyancy force and to the (unknown) pressure force respectively. Differentiation of Eqn. (5.10) gives

$$
u_{1 \zeta \zeta \zeta}-\zeta u_{1 \zeta \xi}=-\theta_{1 \zeta}
$$


For $\xi>0$ a similarity solution can be found for $u_{1 \zeta}^{(\theta)}$ which satisfies $u_{1 \zeta \zeta}^{(\theta)}=-1$ at $\zeta=0$ and $u_{1 \zeta}^{(\theta)} \rightarrow 0$ as $\zeta \rightarrow \infty$ :

$$
\begin{aligned}
u_{1 \zeta}^{(\theta)}=-\frac{3^{1 / 3}}{\Gamma\left(\frac{1}{3}\right)} \zeta \int_{0}^{\zeta / \xi^{1 / 3}} t^{-2} e^{-t^{3} / 9} d t \int_{0}^{t} s e^{(1-\mathrm{Pr}) \mathrm{s}^{3} / 9} d s & \\
& +C_{1} \zeta+C_{2} \zeta \int_{\zeta / \xi^{1 / 3}}^{\infty} t^{-2} e^{-t^{3} / 9} d t
\end{aligned}
$$

where $C_{1}$ and $C_{2}$ are chosen so that the boundary conditions are satisfied. For $\xi<$ $0, \theta_{1}=0$ and so $u_{1}^{(\theta)}=0$. Next $u_{1}^{(p)}$ is found, in terms of the still unknown $p_{1}(\xi)$, by a superposition integral as used by Messiter and $\mathrm{Hu}$ [20] satisfying $u_{1}=0$ and $u_{1 \zeta \zeta}=p_{1}^{\prime}$ at $\zeta=0$ and $u_{1 \zeta} \rightarrow 0$ as $\zeta \rightarrow \infty$ :

$$
u_{1}^{(p)}=-\frac{1}{3^{1 / 3} \Gamma\left(\frac{2}{3}\right)} \int_{0}^{\zeta} d t \int_{-\infty}^{\xi} \frac{p_{1}^{\prime}(s)}{(\xi-s)^{2 / 3}} e^{\left.-(1 / 9) t^{3} / \xi-\dot{s}\right)} d s
$$

Combining Eqns. (A.2) and (A.3) for $\zeta \rightarrow \infty$, we find

$$
u_{1} \sim \frac{3}{2} B \xi^{2 / 3} H(\xi)-\frac{\Gamma\left(\frac{1}{3}\right)}{3^{2 / 3} \Gamma\left(\frac{2}{3}\right)} \int_{-\infty}^{\xi} \frac{p_{1}^{\prime}(s)}{(\xi-s)^{1 / 3}} d s
$$

where $H(\xi)$ is the step function defined by $H(\xi)=0$ for $\xi<0$ and $H(\xi)=1$ for $\xi>0$. The constant $B=B(\operatorname{Pr})$ is obtained by integration of Eqn. (A.2), with $u_{1}^{(\theta)}=$ 0 at $\zeta=0 ; B>0$ since a temperature increase (i.e., $T_{w}^{(d)}>T_{w}^{(u)}$, and so $\lambda<1$ ) accelerates the flow. As $\xi \rightarrow \infty$, the terms in the present formulation should be consistent with Kelleher's [8] downstream solution, obtained for $x \rightarrow 0$. In particular, the solution found for $u_{1}^{(\theta)}$ from Eqn. (A.4) equals $\xi^{2 / 3}$ times a function of $\zeta / \xi^{1 / 3}$ and is proportional to Kelleher's function $f_{1}^{\prime}(\eta)$; the constant $B$ is proportional to $f_{1}^{\prime}(\infty)$.

The right-hand side of Eqn. (A.4) is an expression for the function $G(\xi)$ introduced in Eqn. (5.14). Equating $G^{\prime}(\xi)$ obtained from Eqn. (A.4) with $G^{\prime}(\xi)$ obtained from Eqn. (5.15), we find a relation for $p_{1}(\xi)$ :

$$
\int_{-\infty}^{\xi} p_{1}(s) d s=A \frac{d}{d \xi} \int_{-\infty}^{\xi} \frac{p_{1}^{\prime}(s)}{(\xi-s)^{1 / 3}} d s-B \xi^{-1 / 3} H(\xi)
$$

where $A=3^{-2 / 3} \Gamma\left(\frac{1}{3}\right) / \Gamma\left(\frac{2}{3}\right)$. Thus the temperature changes described by $\theta_{1}(\xi, \zeta)$ can be considered to cause a velocity change given by $u_{1}^{(\theta)}(\xi, \zeta)$. The resulting streamline slope as $\zeta \rightarrow \infty$ is proportional to $\xi^{-1 / 3}$. This leads to a displacement effect for $Y=$ $O(1)$, which in turn requires pressure changes and further velocity changes as described by Eqns. (A.3) and (A.5).

Fourier transformation of Eqn. (A.5) gives

$$
P_{1}(\omega) /(i \omega)=e^{-\pi i / 3} \Gamma\left(\frac{2}{3}\right) \omega^{-2 / 3}\left\{-\omega^{2} A P_{1}(\omega)-B\right\}
$$

where

$$
P_{1}(\omega) \equiv \int_{-\infty}^{\infty} e^{-t \omega \xi} p_{1}(\xi) d \xi
$$


and $\omega$ is complex; we will take $-3 \pi / 2<\arg \omega \leq \pi / 2$. Then

$$
p_{1}(\xi)=-\frac{1}{2 \pi} \frac{B}{A} \int_{-\infty}^{\infty} \frac{\omega^{1 / 3} e^{i \xi \omega} d \omega}{\omega^{7 / 3}-k^{7 / 3} e^{5 \pi i / 6}}
$$

where $k^{7 / 3}=\left[A \Gamma\left(\frac{2}{3}\right)\right]^{-1}=3^{2 / 3} / \Gamma\left(\frac{1}{3}\right)$. For $-3 \pi / 2<\arg \omega \leq \pi / 2$, the singularities of the integrand are simple poles at $\omega / k=e^{5 \pi i / 14}, e^{-\pi i / 2}, e^{-19 \pi i / 14}$. For $\xi<0$, the integral can be evaluated in terms of the residue at $\omega / k=e^{-\pi i / 2}$. For $\xi>0$, Eqn. (A.8) can be replaced by twice the integral along the imaginary axis $\arg \omega=\pi / 2$ plus the contribution from the two poles in the upper half plane. The results are given by Eqns. (5.17) and (5.18).

\section{References}

[1] S. Ostrach, in Theory of Laminar Flows (ed. F. K. Moore), Princeton University Press, 1964.

[2] A. J. EdE, in Advances in Heat Transfer (ed. J. P. Hartnett and T. F. Irvine, Jr.), Vol. 4, Academic Press, New York, 1967, pp. 1-64.

[3] E. Schmidt and W. BeckmanN, Tech. Mech. and Therm. 1, 341-349, 391-406, 1930.

[4] S. Ostrach, NACA TN 2635 (1951); NACA Rep. No. 1111, 1953.

[5] K. T. YANG and E. W. Jerger, J. Heat Transfer 86, 107-115, 1964.

[6] K. T. YANG, J. Appl. Mech. 31 131-138, 1964.

[7] N. E. Hardwick and E. K. Levy, J. Heat Transfer 95, 289-294, 1973.

[8] M. Kelleher, J. Heat Transfer 93, 349-356, 1971.

[9] J. F. Clarke, J. Fluid Mech. 57, 45-61, 1973.

[10] B. Gebhart, J. Fluid Mech. 14, 225-232, 1962.

[11] H. K. KUIKEN, J. Engin. Math. 2, 355-371, 1968.

[12] H. K. Kuiken, J. Fluid Mech. 37, 785-798, 1969.

[13] C.-S. YIH, in Fluid Models in Geophysics (ed. R. R. Long), Government print office, Washington, 1953, pp. 117-133.

[14] S. Goldstein, Proc. Camb. Phil. Soc. 26, 1-30, 1930.

[15] I. ImaI, J. Aero. Sci. 24, 155-156, 1957.

[16] K. Stewartson, Mathematika 16, 106-121, 1969.

[17] A. F. Messiter, SIAM J. Appl. Math. 18, 241-257, 1970.

[18] K. Stewartson and P. G. Williams, Proc. Roy. Soc. A 312, 181-206, 1969.

[19] S. H. SмITH, Mathematika 20, 47-52, 1973.

[20]. A. F. Messiter and J. J. Hu, Q. Appl. Math. 33, 175-181, 1975. 


\section{Zusammenfassung}

Bei laminarer natürlicher Strömung längs einer senkrecht stehenden endlichen geheizten Platte bei sehr grossen Grashof'schen Zahlen gibt es kleine Bereiche, bei denen die Grenzschichtgleichungen keine echte asymptotische Darstellung liefern. Im Vorderkantenbereich findet man ein Zusatzglied zweiter Ordnung für die gesamte Wärmeübertragung. Die Berechnung dieser Korrektur erfolgt durch eine Integralform der Energiegleichung, wobei nur die Lösung der Grenzschichtgleichung zweiter Ordnung fern von der Kante benützt wird, während die Kenntnis der Strömung in der Nähe der Kante nur durch eine Lösung der exakten Navier-Stokes'schen Gleichungen gefunden werden könnte. In der Nähe der Hinterkante und in der Nähe einer Diskontinuität der gegebenen Plattentemperatur wird das Längsdruckgefälle in einer dünnen Unterschicht wichtig; das Querdruckgefälle wird in dem restlichen Teil der Grenzschicht berücksichtigt, für eine Länge, deren Grössenordnung etwas grösser ist, als die der Grenzschichtdicke. Bei der Hinterkante ist das Unterschichtsproblem nicht linear, und es gibt keine analytische Lösung, aber man kann zeigen, dass die Grössenordnung der Korrektur der gesamten Wärmeübertragung etwas grösser ist als die Korrektur bei der Vorderkante. Die Strömung bei der Hinterkante ist dieselbe wie die Strömung bei der Kante einer rotierenden Scheibe in einer sonst ruhenden Flüssigkeit. Das Temperatur-Diskontinuitätsproblem ist linear, und eine Lösung ist hier gefunden worden, wodurch die von der Grenzschichttheorie gegebene Singularität (unendliche Quergeschwindigkeit) entfernt wird.

(Received: April 26, 1976)

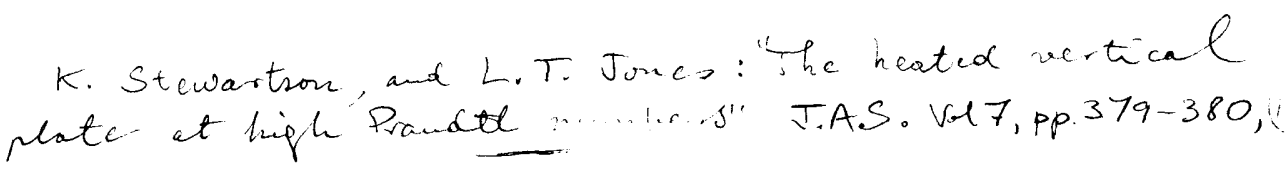

